\title{
Crowdsourcing for Information Retrieval Experimentation and Evaluation
}

\author{
Omar Alonso \\ Microsoft Corp. \\ Mountain View, California, USA \\ omar.alonso@microsoft.com
}

\begin{abstract}
Very recently, crowdsourcing has emerged as a viable alternative for conducting different types of experiments in a wide range of areas. Generally speaking and in the context of IR, crowdsourcing involves outsourcing tasks to a large group of people instead of assigning such tasks to an employee or editor. The availability of commercial crowdsourcing platforms offers vast access to an on-demand workforce. This new approach makes possible to conduct experiments extremely fast, with good results at a low cost. However, like in any experiment, there are several implementation details that would make an experiment work or fail. For large scale evaluation, deployment in practice is not that simple. Tasks have to be designed carefully with special emphasis on the user interface, instructions, content, and quality control.

In this invited talk, I will explore some directions that may influence the outcome of a task and I will present a framework for conducting crowdsourcing experiments making some emphasis on a number of aspects that should be of importance for all sorts of IR-like tasks. Finally, I will outline research trends around human computation that promise to make this emerging field even more interesting in the near future.
\end{abstract}

